\title{
Aplicação do custeio baseado em atividades em uma empresa de serviços rodoviários
}

Mestrado em Administração pela Fundação Universidade Regional de Blumenau -

FURB

Professor da Universidade Estadual do Centro-Oeste - UNICENTRO BR 153 - Km 07. Riozinho, Irati/PR. CEP: 84500-000

E-mail:emacohon@yahoo.com

Jorge Eduardo Scarpin

Doutorado em Controladoria e Contabilidade pela Universidade de São Paulo - USP

Professor da Universidade Federal do Paraná - UFPR Avenida Prefeito Lothário Meissner, 632, 10. andar. Campus III. Jd. Botânico

Curitiba/PR. CEP 80210-170

E-mail: jorge.scarpin@ufpr.br

Willson Gerigk

Doutorado em andamento em Políticas Públicas pela Universidade Federal do Paraná -

UFPR

Professor da Universidade Estadual do Centro-Oeste - UNICENTRO Endereço: BR 153 - Km 07, Riozinho. Irati/PR. CEP: 84500-000

E-mail: gerigkw@gmail.com

Fernanda Castagnolli Domingues

Universidade Estadual do Centro-Oeste - UNICENTRO Rua Agostinho Brusamolin, 333, Bloco 11, Ap. 4. Condomínio Barcelona Neoville. Bairro CIC. Cutitiba/PR. CEP 81312-090

E-mail:fer_castagnolli@hotmail.com

Elvis Fabio Roman Universidade Estadual do Centro-Oeste - UNICENTRO BR 153 - Km 07, Riozinho. Irati/PR. CEP: 84500-000

E-mail:ef.roman@yahoo.com.br

\section{RESUMO}

O estudo tem por objetivo descrever a aplicação do Sistema de Custeio Baseado em Atividades no âmbito de uma Empresa Prestadora de Serviços Rodoviários. Trata-se de uma pesquisa exploratória, realizada por meio de um estudo de caso, com dados coletados através de visitas in loco, privilegiando a abordagem quantitativa. Os resultados apontam as etapas de aplicação do sistema de gestão de custos baseado em atividades, com destaque à identificação dos processos e atividades, levantamento dos recursos consumidos, identificação dos direcionadores de custos e de atividades e 
Aplicação do custeio baseado em atividades em uma empresa de serviços rodoviários Edson Roberto Macohon, Jorge Eduardo Scarpin, Wilson Gerigk, Fernanda Castagnolli Domingues, Elvis

Fabio Roman

rastreamento dos recursos consumidos pelas atividades e estas pelos objetos de custeio. Os processos identificados na prestação de serviços da empresa foram: Conservação da Faixa de Domínio, Sinalização Vertical e Sinalização Horizontal. As atividades identificadas foram: Transporte e Deslocamento, Roçada, Limpeza, Abertura de buracos, Colocação do perfil, Fixação da Placa, Sinalização e Pintura. Na pesquisa foram vislumbrados os seguintes recursos e respectivos direcionadores: Mão de obra, Número de horas (tempo); Combustível, Alocação direta a atividade; Manutenção, Alocação direta a atividade; Depreciação, Alocação direta a atividade; Seguro, Alocação direta a atividade; Materiais, Alocação direta na rodovia (não necessita direcionador). Os resultados apresentados pelo processo de mensuração dos custos realizado reconhecem, primeiramente, os custos a cada uma das atividades e destas para os objetos de custos, as rodovias. A aplicação do sistema de custeamento baseado em atividades na empresa possibilitou conhecer quanto custa a execução dos serviços em cada uma das rodovias, e o mais importante, originou informações do custo de cada processo realizado nessas rodovias.

Palavras-chave: Custeio Baseado em Atividades. Direcionadores de custos. Empresa Prestadora de Serviços Rodoviários.

\section{Application of Activity Based Costing in a Highway Services Company}

\section{ABSTRACT}

The study aims to describe the application of the Activity Based Costing System in the scope of a Highway Services Company. It is an exploratory research, carried out by means of a case study with data collected through on site visits, favoring a quantitative approach. The results show the phases of the implementation of the system of management of costs based on activities, with emphasis on identification of the processes and activities, survey of the resources spent, identification of cost and activities drivers and tracking of the resources spent by the activities, and these by the costing objects. The processes identified in the provision of services of the company were: Conservation of the Area, Vertical Signs and Horizontal Signs. The activities identified were: Transport and Displacement, Land-Clearing, Cleaning, Opening of Holes, Placement of Railings, Sign Mounting, Signalization and Painting. In the research the following resources and contingencies were glimpsed at: Labor, Number of Hours (time), Fuel, Direct Allocation to Activity; Maintenance, Direct Allocation to Activity; Depreciation, Direct Allocation to Activity; Insurance, Direct Allocation to Activity, Materials, Direct Allocation to the Highway (doesn't need a contingent). The results presented by the process of cost measuring recognize, first, the costs to every single activity and from these to the cost objects, the highways. The application of the current expenditures based on the company's activities system allowed to know how much is the cost of implementation of the services in each one of the highways, and most 
Aplicação do custeio baseado em atividades em uma empresa de serviços rodoviários Edson Roberto Macohon, Jorge Eduardo Scarpin, Wilson Gerigk, Fernanda Castagnolli Domingues, Elvis

Fabio Roman

importantly, originated information of the cost of ever single process done in such highways.

Keywords: Activity Based Costing. Costs Drivers. Highway Services Company.

\section{INTRODUÇÃO}

É evidente o crescimento das atividades relacionadas à prestação de serviços no Brasil e exterior. Alguns fatores que contribuíram para a expansão do setor na visão de Perez Jr., Oliveira e Costa (2005) é a crescente informatização dos processos industriais, tendo como consequência a diminuição da participação da mão de obra no setor de transformação e a terceirização de inúmeras atividades secundárias das organizações.

As prestadoras de serviços têm que enfrentar um grande desafio em meio a esse cenário de transformações, precisam se especializar e inovar para sobreviver à demanda por serviços cada vez mais diversificados, clientes cada vez mais exigentes e concorrentes qualificados.

Para tornar-se competitiva é fundamental eliminar os desperdícios e as ineficiências do processo, reduzir custos e, ainda, conseguir manter ou alcançar a excelência nos serviços. No entanto, para atingir tais objetivos é imprescindível a utilização de ferramentas adequadas de gestão de custos. Conforme Gusmão e Almeida (2008), a gestão de custos deve estar voltada para geração de informações que auxiliam a tomada de decisões. A inovação e o gerenciamento eficiente dos custos são fundamentais para a sobrevivência no mercado que se apresenta cada vez mais incerto e competitivo.

O setor de serviços passou a buscar novos conceitos de gerenciamento para acompanhar as exigências do mercado. De acordo com Manuad e Pamplona (2002), a busca de um sistema de custeio que atendesse a esses anseios fez com que as empresas de serviços descobrissem, na manufatura, o Custeio Baseado em Atividades $(A B C)$. O qual se mostrou eficiente e trouxe informações valiosas para o gerenciamento destas organizações. 
Aplicação do custeio baseado em atividades em uma empresa de serviços rodoviários Edson Roberto Macohon, Jorge Eduardo Scarpin, Wilson Gerigk, Fernanda Castagnolli Domingues, Elvis

Com base no exposto, discutem-se as dificuldades enfrentadas pelos gestores em obter informações necessárias para o gerenciamento das organizações e a proposta de aplicação do Sistema de Custeamento por Atividades (ABC) em uma empresa de serviços rodoviários. Assim, com o objetivo de subsidiar respostas a presente discussão, a pesquisa tem o seguinte problema de pesquisa: Como se aplica um sistema de gestão de custos baseado em atividades no âmbito de uma empresa prestadora de serviços rodoviários?

Assim, o objetivo geral do artigo é aplicar o sistema de gestão de custos baseado em atividades no âmbito de uma empresa prestadora de serviços rodoviários. Em consonância com a questão de pesquisa e do objetivo geral, foram propostos os seguintes objetivos específicos: a) descrever os processos e atividades da prestação dos serviços; b) levantar os recursos consumidos na prestação dos serviços; c) identificar os direcionadores dos recursos e das atividades; d) mensurar os custos dos serviços; e) analisar os resultados dos serviços prestados.

Alguns estudos contemplam a utilização do $A B C$ em organizações de serviços, os quais justificam a importância do tema. A pesquisa de Mauad e Pamplona (2002) trata das principais características observadas na implantação do custeio $A B C$ em empresas de serviços. O estudo demonstrou que o Sistema de Custeamento por Atividades provou ser eficiente e trouxe informações mais precisas para o controle dos custos das organizações de serviços pesquisadas.

A pesquisa desenvolvida por Souza et al. (2007) descreve que o ABC proporcionou maior acuracidade no custeamento dos serviços hospitalares. Contudo, revela que o método poderá ter limitações se as organizações não dispuserem de um sistema de informações, infraestrutura em equipamentos de informática e profissionais especializados em gestão financeira e em sistemas de custos.

A relevância do artigo está diante dos problemas que se revelam no âmbito das empresas prestadoras de serviços. Pretende-se contribuir com o aprofundamento das discussões sobre a aplicabilidade do custeio $A B C$ e a disseminação deste sistema em um ramo de atividade pouco pesquisado pela comunidade científica. 
Aplicação do custeio baseado em atividades em uma empresa de serviços rodoviários Edson Roberto Macohon, Jorge Eduardo Scarpin, Wilson Gerigk, Fernanda Castagnolli Domingues, Elvis

Fabio Roman

\section{FUNDAMENTAÇÃO TEÓRICA}

A fundamentação teórica aborda os conceitos utilizados no custeio baseado em atividades $(A B C)$ e discorre sobre o emprego de tal método nas empresas prestadoras de serviços.

\subsection{0 método $A B C$}

O objetivo principal do método ABC é apurar os custos com mais acuracidade. Nakagawa (1994) concebe que o ABC visa rastrear os gastos de uma empresa para analisar e monitorar as diversas rotas de consumo dos recursos diretamente identificáveis com suas atividades mais relevantes, e destas para os produtos e serviços.

Para Bornia (2002, p. 121): "A ideia básica do ABC é tomar os custos das atividades da empresa para entender seu comportamento, encontrando bases que representem as relações entre os produtos e essas atividades". A atribuição mais criteriosa dos custos indiretos por meio das atividades proporciona informações mais detalhadas que permitem ao gestor controlar melhor os gastos da empresa, e também tomar decisões com base em informações mais precisas.

A lógica de apuração dos custos por meio do ABC são as atividades de cada setor da empresa que causam os custos. De acordo com Kaplan e Cooper (1998, p. 99) o enfoque do ABC não é mais alocar os custos: "[...], mas, em primeiro lugar encontrar as razões que justificam o dinheiro gasto pela organização. Ao desenvolver um sistema ABC, a organização identifica inicialmente as atividades que estão sendo executadas por seus recursos indiretos e de apoio".

Conforme Gonçalves e Silva (2007, p. 9), "é muito enganosa a análise de custos que se concentra nos valores e não nos fatores que originam os custos." No entendimento de Perez Jr. e Oliveira (2000), o custeio baseado em atividades parte do princípio de que todos os custos incorridos numa empresa acontecem na execução de uma atividade. 
Aplicação do custeio baseado em atividades em uma empresa de serviços rodoviários Edson Roberto Macohon, Jorge Eduardo Scarpin, Wilson Gerigk, Fernanda Castagnolli Domingues, Elvis

Fabio Roman

Verifica-se que, diferentemente dos métodos tradicionais que consideram que os produtos é que causam os custos, o $A B C$ considera que são as atividades as causadoras dos custos. A essência do $A B C$ são as atividades, são as principais causas dos custos dentro da entidade e sem atividades não existe produção.

\subsubsection{Atividades}

No método de custeio $A B C$ as atividades representam o elemento principal na formação dos custos de produção, visto que é a atividade que consome os recursos para a geração dos bens ou serviços.

Para Ching (2001), uma atividade pode ser definida como aquilo que as pessoas/sistemas fazem. Na concepção de Brimson (1996, p. 62), "uma atividade é uma combinação de pessoas, tecnologia, matérias-primas, métodos e ambiente para gerar determinado produto ou serviço". Martins (2003) explica que o custo de uma atividade compreende todos os sacrifícios de recursos necessários para desempenhála.

No desenvolvimento do $A B C$ as empresam procuram, inicialmente, identificar as atividades executadas (onde foi gasto) que estão consumindo recursos (o que foi gasto), e são descritas por verbos no infinitivo, relacionados com os objetos de custos (produtos, serviços ou clientes), para onde foi gasto (KAPLAN; COOPER, 1998).

Bornia (2002) enfatiza que as atividades devem ser mais detalhadas do que o centro de custos, para que o $A B C$ possa ajudar nas ações de melhoria da empresa. Ching (2001) ressalta que 0 ABC avalia 0 valor que cada atividade agrega para 0 desempenho do negócio ou departamento.

A concepção de criação de valor é também concebida por Maher (2001, p. 315) o qual revela o papel estratégico do método $A B C$ na agregação de valor dos produtos e/ou serviços: 
Aplicação do custeio baseado em atividades em uma empresa de serviços rodoviários Edson Roberto Macohon, Jorge Eduardo Scarpin, Wilson Gerigk, Fernanda Castagnolli Domingues, Elvis

Fabio Roman

Os administradores devem constantemente investigar se as atividades criam valor. [...] devem analisar as atividades e classificá-las em atividades que adicionam valor e atividades que não adicionam valor. As atividades que não adicionam valor devem ser objeto de esforços que visem a sua eliminação, ou, pelo menos, redução.

O reconhecimento das atividades que adicionam ou não valor indica que é necessário repensar o processo de produção ou da prestação de serviços. A percepção dos profissionais de custos é fundamental para o entendimento da lógica do ABC.

\subsubsection{Direcionadores de recursos e de atividades}

$O$ método $A B C$ difere dos métodos tradicionais de custos, principalmente na forma como ele atribui custos à produção. Martins (2003) afirma que o grande desafio, a verdadeira "arte" do $A B C$ está na escolha dos direcionadores de custos. Há que se distinguir dois tipos de direcionadores: os de primeiro estágio, direcionadores de custos de recursos, e os de segundo estágio, chamados de direcionadores de custos de atividades.

O direcionador de recursos, conforme Perez Jr., Oliveira e Costa (2005), é a forma como as atividades consomem os recursos, servindo para custear as atividades, e para demonstrar a relação entre o recurso consumido e as atividades.

Na concepção de Nakagawa (1994, p. 74), um direcionador de custos é: “[...] uma transação que determina a quantidade de trabalho (não a duração) e, através dela, o custo de uma atividade". Ainda afirma que "[...] o cost driver é um evento ou fator causal que influencia o nível e o desempenho de atividades e o consumo resultante de recursos".

Kaplan e Cooper (1998, p. 109) explicam que "[...] a ligação entre atividades e objetos de custo como produtos, serviços e clientes é feita por meio de geradores de custo de atividade. Um gerador de custo de atividade é uma medida quantitativa do resultado de uma atividade". 
Aplicação do custeio baseado em atividades em uma empresa de serviços rodoviários Edson Roberto Macohon, Jorge Eduardo Scarpin, Wilson Gerigk, Fernanda Castagnolli Domingues, Elvis

Fabio Roman

Observa-se que o processo de custeamento do $A B C$ utiliza o conceito de direcionadores para medir e levar os recursos aos objetos de custos. No primeiro estágio os custos gerados são direcionados às atividades que os causaram, por meio de direcionadores de recursos, depois o consumo das atividades é alocado aos produtos e/ou serviços.

\subsection{Custeio baseado em atividade nas empresas de serviços}

Evidencia-se que o método de custeio $A B C$ foi desenvolvido inicialmente para as empresas de manufatura, todavia, observa-se que o método pode ser aplicado com a mesma eficiência nas empresas de serviços.

Embora originário da indústria, o sistema $A B C$ teve desde o início sua aplicação voltada para a área de prestação de serviços (KAPLAN; COOPER, 1998). Souza et al. (2007) afirmam que os custos indiretos (overhead) têm maior participação no custo total do serviço prestado do que na área industrial ou comercial (produto fabricado ou comercializado). Como o sistema $A B C$ concentra sua ação na alocação dos custos indiretos aos produtos e/ou serviços, foi na área da prestação de serviços que mais se difundiu.

Kaplan e Cooper (1998, p. 251) mencionam a aplicabilidade do ABC nas empresas de prestação de serviços:

De modo geral, as empresas de serviços são candidatas ideais ao custeio baseado na atividade, mais ainda que as empresas de produção. Primeiro, praticamente todos os seus custos são indiretos e aparentemente fixos. As empresas de serviços têm volume mínimo ou não têm materiais diretos, a maior parte do seu pessoal oferece suporte indireto, e não direto, a produtos e clientes.

Martins (2003) afirma que as atividades ocorrem tanto em processos de manufatura quanto de prestação de serviços. Portanto é possível utilizar o $A B C$ em instituições financeiras, concessionárias de serviços públicos, telecomunicações, energia e outras prestadoras de serviços. 
Aplicação do custeio baseado em atividades em uma empresa de serviços rodoviários Edson Roberto Macohon, Jorge Eduardo Scarpin, Wilson Gerigk, Fernanda Castagnolli Domingues, Elvis

Fabio Roman

Kaplan e Cooper (1998, p. 249) discorrem sobre as dificuldades de implementação do $A B C$ nas empresas prestadoras de serviços: "As empresas de serviços têm exatamente os mesmos problemas gerenciais enfrentados pelas indústrias. Precisam do custeio baseado na atividade para associar os custos dos recursos que fornecem às receitas geradas pelos produtos e clientes específicos atendidos por esses recursos".

Com a utilização do $A B C$ as empresas de serviços podem aprimorar seus processos internos, identificar as atividades que geram riquezas ou não, e obter os custos de seus serviços de forma mais acurada que os sistemas tradicionais.

\section{MÉTODOS E PROCEDIMENTOS DA PESQUISA}

A presente pesquisa classifica-se como exploratória e foi desenvolvida adotandose o método de estudo de caso. A pesquisa exploratória exposta por Beuren (2006) consiste no aprofundamento de conceitos preliminares sobre determinada temática não contemplada de modo satisfatório anteriormente, contribuindo, deste modo, para o esclarecimento de questões abordadas superficialmente.

Segundo Gil (1999, p. 73), "[..] o estudo de caso é caracterizado pelo estudo profundo e exaustivo de um ou de poucos objetos, de maneira a permitir conhecimentos amplos e detalhados do mesmo, tarefa praticamente impossível mediante outros tipos de delineamento considerados". A pesquisa foi realizada em uma empresa prestadora de serviços de restauração e conservação de rodovias localizada na cidade de Irati/PR no ano de 2011. A adoção do estudo de caso possibilitou uma investigação profunda e detalhada dos processos desenvolvidos e a compreensão de como são incorridos os custos durante as atividades.

A coleta de dados adotou os seguintes procedimentos: entendimento e caracterização dos processos de prestação de serviço; identificação dos elementos de custos e seus respectivos valores; investigação das atividades relevantes relacionadas; análise do consumo de recursos; determinação dos direcionadores de custos e de 
Aplicação do custeio baseado em atividades em uma empresa de serviços rodoviários Edson Roberto Macohon, Jorge Eduardo Scarpin, Wilson Gerigk, Fernanda Castagnolli Domingues, Elvis

Fabio Roman

atividades e o custeamento dos serviços composto por atividades e alocados às rodovias. Os dados coletados tiveram tratamento analítico quantitativo.

O problema da pesquisa requer análises do processo de prestação de serviços da empresa, identificação dos custos, levantamento das atividades e dos direcionadores de custos, bem como avaliação dos resultados alcançados com a utilização do método $A B C$. Desta forma, a pesquisa é classificada como qualitativa quanto a abordagem do problema.

Para Richardson (1999), os estudos que empregam uma metodologia qualitativa podem descrever a complexidade de determinado problema, analisar a interação de certas variáveis, compreender e classificar processos dinâmicos vividos por grupos sociais. Além de contribuir no processo de mudança de determinado grupo e possibilitar, em maior nível de profundidade, o entendimento das particularidades do comportamento dos indivíduos.

\section{DESCRIÇÃO E ANÁLISE DOS RESULTADOS}

Inicialmente apresenta-se a empresa objeto de estudo e a identificação dos serviços executados. Após, aborda-se o reconhecimento das atividades, a identificação dos recursos e respectivos valores, os direcionadores de custos e de atividades, o cálculo dos custos das atividades e dos serviços para cada rodovia e a análise dos resultados obtidos.

\subsection{Apresentação da empresa}

A empresa em estudo é de médio porte, foi constituída em 1998, está sediada na cidade de Irati/PR e atualmente presta serviços de restauração e conservação em rodovias do Paraná que são administradas por concessionárias de pedágio.

\subsection{Identificação dos serviços executados}

O estudo analisou o processo de conservação de rodovias, que compreende três serviços essenciais, conforme apresentado no Quadro 1. 
Aplicação do custeio baseado em atividades em uma empresa de serviços rodoviários Edson Roberto Macohon, Jorge Eduardo Scarpin, Wilson Gerigk, Fernanda Castagnolli Domingues, Elvis

Fabio Roman

\begin{tabular}{|c|c|}
\hline Serviço & Descrição \\
\hline $\begin{array}{c}\text { Conservação } \\
\text { da Faixa de } \\
\text { Domínio (CFD) }\end{array}$ & $\begin{array}{r}\text { - A faixa de domínio compreende a área de cada um dos lados das rodovias, com até } \\
40 \mathrm{~m} \text {. }\end{array}$ \\
$\begin{array}{c}\text { - O proprietário é a União Federal, que repassa para a concessionária a } \\
\text { responsabilidade de administrar essa área junto com a estrada. } \\
\text { seja, preservar as características de acordo com sua concepção original. }\end{array}$ \\
$\begin{array}{c}\text { - Para cumprir com essas exigências a concessionária de pedágios contrata empresas } \\
\text { especializadas para realizar o serviço, consiste basicamente na roçada e limpeza da } \\
\text { área. }\end{array}$ \\
$\begin{array}{c}\text { Sinalização } \\
\text { Vertical (SV) }\end{array}$ & $\begin{array}{c}\text { - É a implantação ou troca das placas que são fixadas ao lado da rodovia, posição } \\
\text { vertical, e transmitem mensagens de regulamentação, advertência e indicação ao } \\
\text { usuário da via. }\end{array}$ \\
\hline $\begin{array}{c}\text { Sinalização } \\
\text { Horizontal (SH) }\end{array}$ & $\begin{array}{c}\text { - É a demarcação realizada sobre as vias, como faixas, marcaçães e símbolos, que } \\
\text { tem a função de organizar e orientar o fluxo de veículos e pedestres. }\end{array}$ \\
\hline
\end{tabular}

Quadro 1 - Serviços executados

Fonte: Dados da pesquisa (2011)

O trecho de rodovias conservadas pela empresa tem 405,9 quilômetros de extensão e abrange cinco rodovias, caracterizadas como: R1 (204 quilômetros), R2 (101 quilômetros), R3 (43 quilômetros), R4 (40,8 quilômetros) e R5 (17,1 quilômetros).

Devido à grande extensão das rodovias e também a distância de algumas à cidade de Irati/PR, a empresa formou três equipes de conservação da faixa de domínio (duas alocadas em outras cidades), com o intuito de diminuir o tempo de transporte até o local de trabalho. Cada equipe é responsável por um determinado trecho das rodovias. O trabalho de cada equipe é contínuo, ou seja, iniciam o trabalho em uma rodovia, realizam a limpeza e roçada diariamente, após iniciam em outra rodovia e assim sucessivamente.

O pessoal que presta serviços de sinalização vertical e horizontal está todo alocado em Irati/PR e é responsável pelo trecho inteiro. $O$ trabalho destas equipes não é contínuo em uma única rodovia, a cada dia possuem rotas distintas de serviços.

\subsection{Reconhecimento das atividades desenvolvidas}

O processo de reconhecimento das atividades foi realizado por meio de entrevistas com os funcionários, a fim de compreender todas as tarefas desempenhadas. Após realizou-se o acompanhamento do trabalho para finalmente agrupar as tarefas e criar o dicionário de atividades, conforme o Quadro 2. 
Aplicação do custeio baseado em atividades em uma empresa de serviços rodoviários Edson Roberto Macohon, Jorge Eduardo Scarpin, Wilson Gerigk, Fernanda Castagnolli Domingues, Elvis

Fabio Roman

\begin{tabular}{|c|c|c|}
\hline Serviço & Atividade & $\begin{array}{l}\text { Descrição das atividades } \\
\end{array}$ \\
\hline $\begin{array}{l}\text { CFD SV } \\
\text { SH }\end{array}$ & $\begin{array}{l}\text { Transporte e } \\
\text { deslocamento }\end{array}$ & $\begin{array}{l}\text { Consiste no deslocamento dos colaboradores até o local de execução } \\
\text { do serviço e no transporte dos equipamentos e materiais que são } \\
\text { utilizados. }\end{array}$ \\
\hline \multirow[b]{2}{*}{ CFD } & Roçada & $\begin{array}{c}\text { É feito o corte da vegetação que cresce sobre a faixa de domínio com a } \\
\text { utilização de roçadeiras mecânicas, com o objetivo de melhorar a } \\
\text { visibilidade da via. }\end{array}$ \\
\hline & Limpeza & $\begin{array}{c}\text { Após o corte da vegetação é feita a limpeza do acostamento, retirando- } \\
\text { se os resíduos (folhas, pedras) que são lançadas no acostamento pelas } \\
\text { máquinas. Para essa atividade é utilizado o soprador, específico para } \\
\text { trabalhos de varrição. }\end{array}$ \\
\hline \multirow{3}{*}{ SV } & $\begin{array}{l}\text { Abertura de } \\
\text { buracos }\end{array}$ & $\begin{array}{l}\text { Com a utilização de ferramentas apropriadas, são cavados os buracos } \\
\text { na profundidade necessária e local solicitado para a implantação da } \\
\text { placa. }\end{array}$ \\
\hline & $\begin{array}{l}\text { Colocação do } \\
\text { perfil }\end{array}$ & O perfil ou "pé da placa" é fixado no local onde a placa será implantada. \\
\hline & Fixação da Placa & $\begin{array}{l}\text { A placa é fixada no perfil com fixadores específicos que o } \\
\text { acompanham. }\end{array}$ \\
\hline \multirow{2}{*}{$\mathrm{SH}$} & Sinalização & $\begin{array}{l}\text { O local onde será realizada a pintura das faixas é sinalizado com cones } \\
\text { e placas nas duas pontas, em cada uma das pontas fica um funcionário } \\
\text { denominado "bandeirinha", função de sinalizar com uma bandeira } \\
\text { vermelha a execução da obra e a orientar o tráfego dos veículos. }\end{array}$ \\
\hline & Pintura & $\begin{array}{l}\text { A pintura das faixas de sinalização é feita com uma máquina própria } \\
\text { para essa atividade, em alguns casos, é feita pintura manual com a } \\
\text { utilização de formas e de uma pistola que é acoplada na máquina. }\end{array}$ \\
\hline
\end{tabular}

Quadro 2 - Dicionário de atividades

Fonte: Dados da pesquisa (2011)

\subsection{Identificação dos recursos consumidos e dos direcionadores}

A definição dos direcionadores requer bastante prudência, a fim de que sejam selecionados os mais adequados, tendo em vista que serão os responsáveis por alocar os custos às atividades e destas para os objetos de custo.

No Quadro 3 estão evidenciados os recursos encontrados que são consumidos pelas atividades durante a execução dos serviços e os direcionadores definidos para alocar os recursos a cada atividade. 
Aplicação do custeio baseado em atividades em uma empresa de serviços rodoviários Edson Roberto Macohon, Jorge Eduardo Scarpin, Wilson Gerigk, Fernanda Castagnolli Domingues, Elvis

Fabio Roman

\begin{tabular}{|c|c|}
\hline Recursos identificados & Direcionador de recurso para a atividade \\
\hline Mão de obra & Número de horas (tempo) \\
\hline Combustível & Alocação direta a atividade \\
\hline Manutenção & Alocação direta a atividade \\
\hline Depreciação & Alocação direta a atividade \\
\hline Seguro & Alocação direta a atividade \\
\hline Materiais & Alocação direta na rodovia (não necessita direcionador) \\
\hline
\end{tabular}

Quadro 3 - Identificação de recursos e respectivos direcionadores

Fonte: Dados da pesquisa (2011)

O gasto com mão de obra foi o único recurso que necessitou de um direcionador, o qual foi definido como o número de horas trabalhadas, uma vez que os funcionários não desempenham uma única atividade.

No Quadro 4 estão elencados os direcionadores definidos para apropriar os custos consumidos pelas atividades aos objetos de custo, ou seja, a cada rodovia.

\begin{tabular}{|c|c|}
\hline Atividade & Direcionador de atividade \\
\hline Transporte e deslocamento & Quilômetros rodados \\
\hline Roçada & Extensão roçada (KM) \\
\hline Limpeza & Extensão da limpeza (KM) \\
\hline Abertura de buracos & Perfis colocados (unidades) \\
\hline Colocação do perfil & Perfis colocados (unidades) \\
\hline Fixação da Placa & Placas fixadas (M²) \\
\hline Sinalização & Tempo (horas) \\
\hline Pintura manual e mecânica & Extensão da pintura (M²) \\
\hline
\end{tabular}

Quadro 4 - Direcionadores de atividade

Fonte: Dados da pesquisa (2011)

Os direcionadores de atividades foram definidos analisando-se a forma como os objetos de custos, no caso as rodovias, consomem essas atividades. Utilizou-se como base para a identificação destes direcionadores a descrição das atividades apresentadas no Quadro 2 (Dicionário de atividades).

\subsection{Cálculo dos custos}

A Tabela 1 revela os valores dos itens de custos consumidos com a conservação de rodovias no mês de março/2011. 
Aplicação do custeio baseado em atividades em uma empresa de serviços rodoviários Edson Roberto Macohon, Jorge Eduardo Scarpin, Wilson Gerigk, Fernanda Castagnolli Domingues, Elvis

Fabio Roman

Tabela 1 - Custos da conservação de rodovias

\begin{tabular}{|c|c|}
\hline Recurso & Valor $\mathbf{R} \mathbf{\$}$ \\
\hline Mão de obra & $108.306,12$ \\
\hline Combustível & $22.389,47$ \\
\hline Manutenção & $11.813,09$ \\
\hline Depreciação & $13.217,32$ \\
\hline Seguro & $1.092,00$ \\
\hline Material & $142.486,11$ \\
\hline Total & $\mathbf{2 9 9 . 3 0 4 , 1 1}$ \\
\hline
\end{tabular}

Fonte: Dados da pesquisa (2011)

Ressalta-se que o item "mão de obra" abrange os valores de salários e horas extras com respectivos encargos; provisões de férias e $13^{\circ}$ salário com respectivos encargos; e assistências (médica, odontológica, alimentação e seguro de vida).

Os valores de combustível, manutenção, depreciação e seguro compreendem todos os custos demandados com o funcionamento e desgaste dos veículos e equipamentos. O valor do material consumido é composto por placas, perfis (utilizados na Sinalização Vertical) tintas, solventes e microesferas de vidro (utilizados na Sinalização Horizontal).

A Tabela 2 apresenta o cálculo do custo do serviço de manutenção da faixa e domínio de acordo com os direcionadores estabelecidos no Quadro 3.

Tabela 2 - Custo do serviço de manutenção da faixa e domínio

\begin{tabular}{|c|c|c|c|c|c|c|c|c|}
\hline \multirow{3}{*}{ Recurso } & \multicolumn{6}{|c|}{ Atividades } & \multirow{3}{*}{$\begin{array}{c}\text { Total } \\
\text { recurso }\end{array}$} & \multirow{3}{*}{$\%$} \\
\hline & \multicolumn{2}{|c|}{ Transporte } & \multicolumn{2}{|c|}{ Roçada } & \multicolumn{2}{|c|}{ Limpeza } & & \\
\hline & $\mathbf{R} \$$ & & $\mathbf{R} \$$ & & $\mathbf{R} \$$ & & & \\
\hline Mão de obra & $2.470,07$ & $15 \%$ & $72.093,35$ & $3 \%$ & $17.078,10$ & $90 \%$ & 91.641 .52 & $75 \%$ \\
\hline Combustível & $5.970,51$ & $37 \%$ & $9.776,27$ & $11 \%$ & $1.335,56$ & $7 \%$ & $17.082,34$ & $14 \%$ \\
\hline Manutenção & $5.382,46$ & $33 \%$ & $1.758,16$ & $2 \%$ & 38,00 & $0 \%$ & $7.178,62$ & $6 \%$ \\
\hline Depreciação & $1.791,67$ & $11 \%$ & $3.249,75$ & $4 \%$ & 466,67 & $2 \%$ & $5.508,09$ & $4 \%$ \\
\hline Seguro & 512,62 & $3 \%$ & & $0 \%$ & & $0 \%$ & 512,62 & $1 \%$ \\
\hline Custo atividade & $16.127,33$ & $3 \%$ & $86.877,53$ & $1 \%$ & $18.918,33$ & $6 \%$ & $121.923,19$ & $100 \%$ \\
\hline Custo serviço & & & & & & & $121.923,19$ & $100 \%$ \\
\hline
\end{tabular}

Fonte: Dados da pesquisa (2011) 
Aplicação do custeio baseado em atividades em uma empresa de serviços rodoviários Edson Roberto Macohon, Jorge Eduardo Scarpin, Wilson Gerigk, Fernanda Castagnolli Domingues, Elvis

Fabio Roman

O custo com a manutenção da faixa de domínio foi de $R \$ 121.923,19$, este valor é composto principalmente por mão de obra (75\%).

A Tabela 3 evidencia o cálculo do custo do serviço de sinalização vertical de acordo com os direcionadores estabelecidos no Quadro 3.

Tabela 3 - Custo do serviço de sinalização vertical

\begin{tabular}{|c|c|c|c|c|c|c|c|c|c|c|}
\hline \multirow{3}{*}{ Recurso } & \multicolumn{8}{|c|}{ Atividades } & \multirow{3}{*}{$\begin{array}{l}\text { Total } \\
\text { recurso }\end{array}$} & \\
\hline & \multicolumn{2}{|c|}{ Transporte } & \multicolumn{2}{|c|}{$\begin{array}{l}\text { Abertura } \\
\text { buracos }\end{array}$} & \multicolumn{2}{|c|}{$\begin{array}{c}\text { Colocação } \\
\text { perfil }\end{array}$} & \multicolumn{2}{|c|}{$\begin{array}{l}\text { Fixação da } \\
\text { placa }\end{array}$} & & \\
\hline & $\mathbf{R} \mathbf{S}$ & & $\mathbf{R} \$$ & & $\mathbf{R} \$$ & & $\mathbf{R} \mathbf{\$}$ & & & \\
\hline Mão de obra & 715,16 & 7 & $3.698,24$ & 100 & $1.895,90$ & 100 & $1.931,67$ & 00 & $8.240,97$ & 45 \\
\hline Combustível & $3.016,84$ & 28 & & & & & & & $3.016,84$ & 17 \\
\hline Manutenção & 848,34 & 8 & & & & & & & 848,34 & 5 \\
\hline Depreciação & $5.666,67$ & 53 & & & & & & & $5.666,67$ & 31 \\
\hline Seguro & 421,31 & 4 & & & & & & & 421,31 & 2 \\
\hline $\begin{array}{c}\text { Custo } \\
\text { atividade }\end{array}$ & $10.668,32$ & 59 & $3.698,24$ & 20 & $1.895,90$ & 0 & $1.931,67$ & 1 & $18.194,13$ & 14 \\
\hline Material & & & & & & & & & $110.183,93$ & 86 \\
\hline $\begin{array}{l}\text { Custo } \\
\text { serviço }\end{array}$ & & & & & & & & & $128.378,06$ & 100 \\
\hline
\end{tabular}

Fonte: Dados da pesquisa (2011)

O serviço de sinalização vertical teve um custo total de $R \$ 128.378,93$, sendo representado por $R \$ 18.194,13$ (14\%) de custos indiretos consumidos pelas atividades e $\mathrm{R} \$ 110.183,93$ (86\%) de materiais. Considerando somente os custos das atividades, observa-se que 59\% são causados com a atividade de transporte, e que deste valor a depreciação tem a maior representatividade $53 \%$. As demais atividades utilizam apenas mão de obra em sua execução.

A Tabela 4 mostra o cálculo do custo do serviço de sinalização horizontal de acordo com os direcionadores estabelecidos no estudo (Quadro 3). 
Aplicação do custeio baseado em atividades em uma empresa de serviços rodoviários Edson Roberto Macohon, Jorge Eduardo Scarpin, Wilson Gerigk, Fernanda Castagnolli Domingues, Elvis

Tabela 4 - Custo do serviço de sinalização horizontal

\begin{tabular}{|c|c|c|c|c|c|c|c|c|}
\hline \multirow{3}{*}{ Recurso } & \multicolumn{6}{|c|}{ Atividades } & \multirow{3}{*}{ Total recurso } & \\
\hline & \multicolumn{2}{|c|}{ Transporte } & \multicolumn{2}{|c|}{ Abertura buracos } & \multicolumn{2}{|c|}{ Colocação perfil } & & \\
\hline & $\mathbf{R} \$$ & & $\mathbf{R} \$$ & & $\mathbf{R} \$$ & & & \\
\hline Mão de obra & $1.688,87$ & 22 & $3.026,79$ & 100 & $3.707,97$ & 62 & $8.423,63$ & 50 \\
\hline Combustível & $2.010,86$ & 26 & & & 279,43 & 5 & $2.290,29$ & 14 \\
\hline Manutenção & $3.463,63$ & 45 & & & 322,50 & 5 & $3.786,13$ & 23 \\
\hline Depreciação & 459,23 & 6 & & & $1.583,33$ & 26 & $2.042,56$ & 12 \\
\hline Seguro & 68,72 & 1 & & & 89,35 & 1 & 158,07 & 1 \\
\hline $\begin{array}{c}\text { Custo } \\
\text { atividade }\end{array}$ & $7.691,31$ & 46 & $3.026,79$ & 8 & $5.982,58$ & 36 & $16.700,68$ & 34 \\
\hline Materiais & & & & & & & $32.302,18$ & 66 \\
\hline Custo serviço & & & & & & & $49.002,86$ & 100 \\
\hline
\end{tabular}

Fonte: Dados da pesquisa (2011)

O serviço de sinalização horizontal custou $R \$ 49.002,86$, 34\% de custos indiretos e $66 \%$ de custos com materiais. O custo com atividades é composto com $50 \%$ de mão de obra e outros $50 \%$ o valor gasto com as máquinas e equipamentos. $A$ atividade de transporte consome a maior parte dos custos $46 \%$, a atividade de pintura $36 \%$. A Tabela 5 apresenta os custos dos serviços de conservação da faixa de domínio.

Tabela 5 - Custo do serviço de conservação da faixa de domínio por rodovia

\begin{tabular}{|c|c|c|c|c|c|c|c|c|c|}
\hline Direcionador & \multicolumn{2}{|c|}{$\mathrm{KM}$ rodados } & \multicolumn{2}{|c|}{ KM roçados } & \multicolumn{2}{|c|}{ KM limpeza } & \multirow{2}{*}{$\begin{array}{l}\text { Custo por } \\
\text { rodovia }\end{array}$} & \multirow{2}{*}{$\%$} & \multirow{2}{*}{$\begin{array}{c}\text { Custo } \\
\mathrm{KM} \\
\text { roçado }\end{array}$} \\
\hline Atividade & Transporte & $\%$ & Roçada & $\%$ & Limpeza & $\%$ & & & \\
\hline \multirow{2}{*}{ Rod. 1} & 5.898 & & 189,6 & & 189,6 & & - & & \\
\hline & $9.085,78$ & 56 & $46.596,83$ & 54 & $10.146,86$ & 4 & $65.829,47$ & 54 & 347,20 \\
\hline \multirow{2}{*}{ Rod. 2} & 2.409 & & 92,3 & & 92,3 & & - & & \\
\hline & $3.711,03$ & 23 & $22.684,01$ & 26 & $4.939,64$ & 26 & $31.334,67$ & 26 & 339,49 \\
\hline \multirow{2}{*}{ Rod. 3} & 1.290 & & 46,0 & & 46,0 & & - & & \\
\hline & $1.987,22$ & 12 & $11.305,14$ & 13 & $2.461,79$ & 13 & $15.754,15$ & 13 & 342,48 \\
\hline \multirow{2}{*}{ Rod. 4} & 683 & & 18,6 & & 18,6 & & - & & \\
\hline & $1.052,15$ & 7 & $4.571,21$ & 5 & 995,42 & 5 & $6.618,78$ & 5 & 355,85 \\
\hline \multirow{2}{*}{ Rod. 5} & 189 & & 7,0 & & 7,0 & & - & & \\
\hline & 291,15 & 2 & $1.720,35$ & 2 & 374,62 & 2 & $2.386,12$ & 2 & 340,87 \\
\hline $\begin{array}{c}\text { Soma } \\
\text { direcionadores }\end{array}$ & 10.469 & & 353,5 & & 353,5 & & - & & \\
\hline Custo atividade & $16.127,33$ & 100 & $86.877,53$ & 100 & $18.918,33$ & 100 & $121.923,19$ & 100 & 344,90 \\
\hline
\end{tabular}

Fonte: Dados da pesquisa (2011) 
Aplicação do custeio baseado em atividades em uma empresa de serviços rodoviários Edson Roberto Macohon, Jorge Eduardo Scarpin, Wilson Gerigk, Fernanda Castagnolli Domingues, Elvis

Fabio Roman

Durante o mês foram limpos $353,5 \mathrm{~km}$ de rodovias, os veículos de transporte percorreram $10.469 \mathrm{~km}$. A Tabela 6 demonstra a alocação do custo das atividades de sinalização vertical.

Tabela 6 - Custo do serviço de sinalização vertical por rodovia

\begin{tabular}{|c|c|c|c|c|c|c|c|c|c|c|c|c|c|}
\hline \multirow{2}{*}{$\begin{array}{l}\text { Direc. } \\
\text { Ativ. }\end{array}$} & \multicolumn{2}{|c|}{$\mathrm{KM}$ rodados } & \multicolumn{2}{|c|}{ № perfis } & \multicolumn{2}{|c|}{ № perfis } & \multicolumn{2}{|c|}{$\mathbf{M}^{2}$ placa } & \multirow[b]{2}{*}{ Mat. } & \multirow[b]{2}{*}{$\%$} & \multirow[b]{2}{*}{$\begin{array}{c}\text { Custo } \\
\text { rod. }\end{array}$} & \multirow[b]{2}{*}{$\%$} & \multirow{2}{*}{$\begin{array}{c}\text { Custo } \\
\mathbf{M}^{2} \\
\text { placa }\end{array}$} \\
\hline & $\begin{array}{c}\text { Tranp. } \\
\text { desl. }\end{array}$ & $\%$ & $\begin{array}{c}\text { Abert. } \\
\text { bur. }\end{array}$ & $\%$ & $\begin{array}{c}\text { Fix. } \\
\text { Perfis }\end{array}$ & $\%$ & $\begin{array}{c}\text { Fix. } \\
\text { Placa } \\
\text { s } \\
\end{array}$ & $\%$ & & & & & \\
\hline \multirow{2}{*}{ Rod. 1} & 2.406 & & 146 & & 146 & & 183 & & & & - & & \\
\hline & 5.462 & 51 & 2.421 & 65 & 1.241 & 65 & 1.384 & 72 & 80.218 & 73 & 90.728 & 71 & 495,19 \\
\hline \multirow{2}{*}{ Rod. 2} & 1.526 & & 48 & & 48 & & 45,34 & & & & - & & \\
\hline & 3.464 & 32 & 796 & 22 & 408 & 22 & 342 & 18 & 19.062 & 17 & 24.073 & 19 & 530,96 \\
\hline \multirow{2}{*}{ Rod. 3} & 486 & & 2 & & 2 & & 2,00 & & & & - & & \\
\hline & 1.103 & 10 & 33 & 1 & 17 & 1 & 15 & 1 & 816 & 1 & 1.985 & 2 & 992,80 \\
\hline \multirow{2}{*}{ Rod. 4} & 227 & & 2 & & 2 & & 2,00 & & & & - & & \\
\hline & 515 & 5 & 33 & 1 & 17 & 1 & 15 & 1 & 816 & 1 & 1.397 & 1 & 698,79 \\
\hline \multirow{2}{*}{ Rod. 5} & 54 & & 25 & & 25 & & 22,98 & & & & - & & \\
\hline & 122 & 1 & 414 & 11 & 212 & 11 & 173 & 9 & 9.268 & 8 & 10.192 & 8 & 403,34 \\
\hline Soma & 4.699 & & 223 & & 223 & & 255 & & & & - & & \\
\hline $\begin{array}{c}\text { Custo } \\
\text { ativ. }\end{array}$ & 10.668 & 100 & 3.698 & 100 & 1.895 & 100 & 1.931 & 100 & 110.183 & 100 & 128.378 & 100 & 502,38 \\
\hline
\end{tabular}

Fonte: Dados da pesquisa (2011)

Durante o mês foram instalados nas rodovias 255,54 metros quadrados de placa e 223 perfis, para realizar o serviço, o veículo utilizado no transporte percorreu 4.699 $\mathrm{km}$. A demanda do serviço foi maior nas rodovias 1 e 2 . A primeira recebeu a implantação de $72 \%$ das placas e $65 \%$ dos perfis colocados no mês, na rodovia 2 foram instalados $18 \%$ do total de placas e $22 \%$ dos perfis. Ressalta-se a grande diferença nos percentuais de apropriação de custos da atividade de transporte em relação às demais atividades.

Essa diferença explica-se pelo fato de ser uma única equipe localizada na cidade de Irati/PR para executar o serviço em todas as rodovias, sendo assim as rodovias mais distantes recebem uma parcela maior dos custos. Essa situação pode ser visualizada com clareza nas rodovias 3 e 4, que a quantidade de placas e perfis implantados são iguais, porém o valor do custo atribuído para as duas rodovias é diferente. Para 
Aplicação do custeio baseado em atividades em uma empresa de serviços rodoviários Edson Roberto Macohon, Jorge Eduardo Scarpin, Wilson Gerigk, Fernanda Castagnolli Domingues, Elvis

Fabio Roman

executar o serviço na rodovia 3 foi necessário percorrer $486 \mathrm{~km}$ e na rodovia 4, apenas $227 \mathrm{~km}$.

Tal situação causa grande variação no custo unitário. O custo unitário médio utilizando-se a medida das placas fixadas foi de $R \$ 502,38$, a Rodovia 3 teve um custo unitário de $\mathrm{R} \$ 992,80$.

$\mathrm{Na}$ Tabela 7, apresenta-se a distribuição do custo das atividades do serviço de sinalização horizontal, conforme os direcionadores de custos estabelecidos no Quadro 4.

Tabela 7 - Custo do serviço de sinalização horizontal por rodovia

\begin{tabular}{|c|c|c|c|c|c|c|c|c|c|c|c|}
\hline Direc. & \multicolumn{2}{|c|}{ KM rodados } & \multicolumn{2}{|l|}{ Horas } & \multicolumn{2}{|c|}{$M^{2}$ pintura } & \multirow[b]{2}{*}{ Material } & \multirow[b]{2}{*}{$\%$} & \multirow{2}{*}{$\begin{array}{l}\text { Custo } \\
\text { por } \\
\text { rodovia }\end{array}$} & \multirow[b]{2}{*}{$\%$} & \multirow{2}{*}{$\begin{array}{c}\text { Custo } \\
M^{2} \\
\text { pintura }\end{array}$} \\
\hline Atividade & Transporte & $\%$ & Sinalização & $\%$ & Pintura & $\%$ & & & & & \\
\hline \multirow{2}{*}{ Rod. 1} & 1.193 & & 90,8 & & 4395,5 & & & & - & & \\
\hline & $2.633,68$ & 34 & $1.906,44$ & 63 & $3.572,30$ & 60 & $19.995,76$ & 62 & $28.108,18$ & 57 & 6,39 \\
\hline \multirow{2}{*}{ Rod. 2} & 1.238 & & 31,92 & & 2081,3 & & & & - & & \\
\hline & $2.733,02$ & 36 & 670,19 & 22 & $1.691,51$ & 28 & $8.147,95$ & 25 & $13.242,67$ & 27 & 6,36 \\
\hline \multirow{2}{*}{ Rod. 3} & 820 & & 16,96 & & 526,2 & & & & - & & \\
\hline & $1.810,24$ & 24 & 356,09 & 12 & 427,65 & 7 & $2.229,20$ & 7 & $4.823,18$ & 10 & 9,17 \\
\hline \multirow{2}{*}{ Rod. 4} & 233 & & 4,48 & & 358,2 & & & & - & & \\
\hline & 514,37 & 7 & 94,06 & 3 & 291,12 & 5 & $1.929,28$ & 6 & $2.828,83$ & 6 & 7,90 \\
\hline \multirow{2}{*}{ Rod. 5} & 0 & & 0 & & 0 & & & & - & & \\
\hline & 0,00 & 0 & 0,00 & 0 & 0,00 & 0 & & 0 & 0,00 & 0 & \\
\hline Soma & 3.484 & & 144 & & 7.361 & & & & - & & \\
\hline $\begin{array}{l}\text { Custo } \\
\text { Ativid. }\end{array}$ & $7.691,31$ & 100 & $3.026,79$ & 100 & $5.982,58$ & 100 & $32.302,19$ & 100 & $49.002,87$ & 100 & 6,66 \\
\hline
\end{tabular}

Fonte: Dados da pesquisa (2011)

Foram realizados 7.361 metros quadrados de pintura nas rodovias, sendo que $60 \%$ foi realizada na Rodovia 1 e $28 \%$ na Rodovia 2. No entanto, o custo com a atividade de transporte foi maior na Rodovia 2. Na Rodovia 3, o percentual de apropriação de custo da atividade de transporte foi bastante superior a apropriação das outras atividades.

A mesma situação verificada no serviço de sinalização vertical com relação à atividade de transporte ocorre, também nos serviços de sinalização horizontal. $\mathrm{Na}$ rodovia 3 , por exemplo, os custos apropriados na atividade de pintura são de $7 \%$ e na 
Aplicação do custeio baseado em atividades em uma empresa de serviços rodoviários Edson Roberto Macohon, Jorge Eduardo Scarpin, Wilson Gerigk, Fernanda Castagnolli Domingues, Elvis

atividade de transporte $24 \%$, com essa situação tem-se um custo por metro quadrado de pintura de $R \$ 9,17$, sendo que o custo médio é de $R \$ 6,66$.

\subsection{Análise dos resultados obtidos com a aplicação do ABC}

Atualmente, a empresa faz a distribuição dos custos indiretos para as rodovias utilizando apenas a medição dos serviços como critério de rateio. A utilização desse único critério gera a apropriação desses custos de forma arbitrária, gerando com isso informações distorcidas sobre os custos dos serviços executados.

A apropriação dos custos das atividades para as rodovias com a utilização dos direcionadores, permite uma visualização detalhada do consumo de recursos em cada um dos serviços. Os dados encontrados com a utilização do $A B C$ indicam que a empresa deve ter uma atenção especial no processo de custeamento, considerar o custo com o deslocamento na execução dos serviços em cada rodovia, pois, é um fator que afeta o custo unitário e, consequentemente, o preço do serviço.

Conforme observado anteriormente, algumas rodovias recebem uma proporção de custo da atividade de transporte muito divergente da proporção alocada das outras atividades, impactando o custo unitário do serviço de cada rodovia. Isso ocorre nos serviços de sinalização vertical e horizontal, os funcionários estão alocados em uma única cidade, necessitando percorrer grandes distâncias para realizar um serviço que não demanda muito tempo, como por exemplo, fixação de duas placas.

$\mathrm{Na}$ conservação da faixa de domínio a atividade de roçada consumiu $71 \%$ dos custos com o serviço. Já na sinalização horizontal e vertical não foram as atividades fim que utilizaram a maior parte dos custos indiretos, mas sim a atividade de transporte e deslocamento, que é uma atividade meio, necessária para a execução das demais.

\section{CONCLUSÃO}

Realizou-se uma pesquisa exploratória, por meio de um estudo de caso com o objetivo principal de descrever a aplicação de um sistema de gestão de custos baseado em atividades no âmbito de uma empresa prestadora de serviços rodoviários. 
Aplicação do custeio baseado em atividades em uma empresa de serviços rodoviários Edson Roberto Macohon, Jorge Eduardo Scarpin, Wilson Gerigk, Fernanda Castagnolli Domingues, Elvis

Fabio Roman

Os processos identificados na prestação de serviços da empresa foram: Conservação da Faixa de Domínio, Sinalização Vertical e Sinalização Horizontal. As atividades identificadas foram: Transporte e Deslocamento, Roçada, Limpeza, Abertura de buracos, Colocação do perfil, Fixação da Placa, Sinalização e Pintura.

$\mathrm{Na}$ pesquisa foram vislumbrados os seguintes recursos e respectivos direcionadores: Mão de obra, Número de horas (tempo); Combustível, Alocação direta a atividade; Manutenção, Alocação direta a atividade; Depreciação, Alocação direta a atividade; Seguro, Alocação direta a atividade; Materiais, Alocação direta na rodovia (não necessita direcionador).

A seleção dos direcionadores foi realizada por meio das rotas do consumo dos recursos. No entanto, alguns recursos consumidos não necessitaram de direcionador, pois foram facilmente identificados às atividades, devido a controles já realizados pela empresa.

Os custos identificados nas atividades foram alocados aos objetos de custos por meio dos direcionadores de atividades. Estes direcionadores foram definidos analisando-se a forma como os objetos de custos, no caso as rodovias, consomem essas atividades.

Por fim, os resultados apresentados pelo processo de mensuração dos custos realizado reconhecem, primeiramente, os custos a cada uma das atividades e destas para os objetos de custos, as rodovias. A aplicação do sistema de custeamento baseado em atividades na empresa possibilitou conhecer quanto custa a execução dos serviços em cada uma das rodovias, e o mais importante, originou informações do custo de cada processo realizado nessas rodovias.

Ressalta-se alguns pontos favoráveis da empresa que facilitaram à aplicação do sistema de gestão de custos baseado em atividades, a disponibilidade de um sistema de informações que auxilia e gera relatórios dos gastos de veículos e equipamentos; e, são realizados controles efetivos da prestação dos serviços, bem como dos materiais utilizados. 
Aplicação do custeio baseado em atividades em uma empresa de serviços rodoviários Edson Roberto Macohon, Jorge Eduardo Scarpin, Wilson Gerigk, Fernanda Castagnolli Domingues, Elvis

Fabio Roman

A principal dificuldade encontrada foi a mensuração das horas consumidas pelos colaboradores na realização das atividades, visto que não executam trabalhos diversificados.

Conclui-se que foi perfeitamente possível aplicar o sistema de gestão de custos baseado em atividades no âmbito de uma empresa prestadora de serviços rodoviários e recomenda-se a utilização desta ferramenta na gestão de custos em organizações de serviços.

\section{REFERÊNCIAS}

BEUREN, I. M. 2006. Como elaborar trabalhos monográficos em contabilidade: teoria e prática. (3 ed.). São Paulo: Atlas.

BORNIA, A. C. 2002. Análise gerencial de custos em empresas modernas. Porto Alegre: Bookman.

BRIMSON, J. A. 1996. Contabilidade por atividades: uma abordagem de custeio baseado em atividades. Tradução de Antonio T. G. Carneiro. São Paulo: Atlas.

CHING, H. Y. 2001. Gestão baseada em custeio por atividades: activity based management. (3 ed.). São Paulo: Atlas.

GONÇALVES, R. C. M. G.; SILVA, A. C. 2007. A qualidade da informação de custo considerando-se diferentes necessidades e diferentes métodos de custeio. Revista Pensar Contábil. Vol. 9, N. 35, ISSN 2177-417X.

GIL, A. C. 1999. Métodos e técnicas de pesquisa social. (5 ed.). São Paulo: Atlas.

GUSMÃO, I. B., ALMEIDA, L. B. 2008. A utilização dos custos no suporte as atividades de marketing: um estudo no setor hoteleiro de Curitiba e região metropolitana. In: Congresso ANPCONT, 2. Associação Nacional dos Programas de Pós-Graduação em Ciências Contábeis, Salvador. Disponível em:

https://www.furb.br/especiais/download/568559-223145/ccg\%20132\%20-

\%20index3.php.pdf. Acesso em: 10/ago/2011.

KAPLAN, R. S.; COOPER, R. 1998. Custo e desempenho: administre seus custos para ser mais competitivo. São Paulo: Futura.

MARTINS, E.2003. Contabilidade de custos. (9 ed.). São Paulo: Atlas. 
Aplicação do custeio baseado em atividades em uma empresa de serviços rodoviários Edson Roberto Macohon, Jorge Eduardo Scarpin, Wilson Gerigk, Fernanda Castagnolli Domingues, Elvis

Fabio Roman

MAUAD, L. G. A.; PAMPLONA, E. O. 2001. O custeio abc em empresas de serviços: avaliação dos problemas da implantação em uma empresa de pesquisa. VIII ABCustos, São Leopoldo. Disponível em: http://www.iepg.unifei.edu.br/ edson/download/Artguilacongcustos01.pdf. Acesso em: 10/ago/2011.

. 2002. O custeio abc em empresas de serviços: características observadas na implantação em uma empresa do setor. IX Congresso Brasileiro de Custos, São Paulo. Disponível em: <http://www.iem.efei.br>. Acesso em: 12/jul/2011.

NAKAGAWA, M. Abc - custeio baseado em atividades. São Paulo: Atlas, 1994.

PEREZ JR, J. H., OLIVEIRA, L. M. 2000. Contabilidade para não contadores. São Paulo: Atlas.

Atlas.

; COSTA, R. G. 2005. Gestão estratégica de custos. (4 ed.). São Paulo:

RICHARDSON, R. J. 1999. (org) et al. Pesquisa social: métodos e técnicas. São Paulo: Atlas.

SOUZA, A. A. et al. 2007. Uma aplicação do custeio baseado em atividades na central de materiais esterilizados de hospitais. In: Congresso Brasileiro de Custos, João Pessoa, Paraíba: ABC, FEA/USP, 2007. Cd-Rom.

Data de Submissão: 16/06/2012

Data de Aceite: 01/10/2015 